\title{
Generalized Lyapunov exponents of the random harmonic oscillator: cumulant expansion approach
}

\author{
Raúl O. Vallejos 1 , 田 and Celia Anteneodo2, 团 \\ ${ }^{1}$ Centro Brasileiro de Pesquisas Físicas (CBPF), \\ Rua Dr. Xavier Sigaud 150, 22290-180 Rio de Janeiro, Brazil \\ ${ }^{2}$ Department of Physics, PUC-Rio and National Institute of Science and Technology for Complex Systems, \\ Rua Marquês de São Vicente 225, Gávea, CEP 22453-900 RJ, Rio de Janeiro, Brazil
}

(Dated: November 7, 2018)

\begin{abstract}
The cumulant expansion is used to estimate generalized Lyapunov exponents of the randomfrequency harmonic oscillator. Three stochastic processes are considered: Gaussian white noise, Ornstein-Uhlenbeck, and Poisson shot noise. In some cases, nontrivial numerical difficulties arise. These are mostly solved by implementing an appropriate importance-sampling Montecarlo scheme. We analyze the relation between random-frequency oscillators and many-particle systems with pairwise interactions like the Lennard-Jones gas.
\end{abstract}

PACS numbers: 05.45.-a, 05.40.-a, 02.50.Ey

\section{INTRODUCTION}

Lyapunov exponents quantify sensitivity to initial conditions in dynamical systems. The existence of a positive Lyapunov exponent implies that trajectories initially close in phase space will typically diverge exponentially fast in time. In practice, this sets a limit for predicting the future behavior of the system, because small imprecisions in the knowledge of the initial state will be amplified at a rate given by the largest Lyapunov exponent. Even if determinism subsists on a short time scale, on longer time windows the system exhibits features of randomness. This property lies at the basis of the statistical description of many-particle deterministic systems. Hence the interest in analytical estimates of Lyapunov exponents in simple statistical-mechanics models.

The theory of Lyapunov exponents of hard-ball systems has a long history. It started with the pioneering work of Krylov [1, 2], was rigorously developed by Sinai [3] and collaborators, and completed (to some extent) by van Beijeren, Dorfman and co-workers [4 8]. The analytical calculation of, e.g., the largest Lyapunov exponent of a dilute rigid-sphere gas, is based on the fact that the dynamics consists of free rectilinear motions interrupted by instantaneous elastic collisions [6]; the expressions soobtained agree quantitatively with the numerical experiments [6, 9, 10].

The case of a dilute gas with finite-range interactions can be handled in close analogy with the rigid-sphere problem: though the collisions are not trivial any more, the dynamics is still ruled by occasional pairwise encounters $[6,11,12$. However, when one considers long-range interactions (or short-range interactions and high densities), the theoretical approach must be substantially

\footnotetext{
*Electronic address:

URL: http://www.cbpf.br/ vallejos

${ }^{\dagger}$ Electronic address: celia@fis.puc-rio.br
}

modified.

In the general case we must deal with the full system of coupled differential equations that govern the evolution of multidimensional tangent vectors $\eta(t)$. Consider for instance a gas of $N$ particles in three dimensions described by the Hamiltonian

$$
\mathcal{H}=\sum_{i=1}^{3 N} \frac{p_{i}^{2}}{2 m}+\mathcal{V}\left(q_{1}, \ldots, q_{3 N}\right),
$$

where $q_{i}$ and $p_{i}$, are conjugate position-momentum coordinates. Assuming $m=1$, tangent vectors evolve according to

$$
\dot{\eta}=\left(\begin{array}{cc}
0 & 1 \\
-\mathbf{V}(t) & 0
\end{array}\right) \eta
$$

(dot meaning time derivative), where $\mathbf{V}$ is the Hessian matrix of the potential $\mathcal{V}$, namely

$$
V_{i j}=\frac{\partial^{2} \mathcal{V}}{\partial q_{i} \partial q_{j}}
$$

The Hessian depends explicitly on time because it is calculated along a reference trajectory $q(t)$. Once initial conditions $z_{0}=\left(q_{0}, p_{0}\right)$ and $\eta_{0}$ have been specified, one can find $\eta(t)$ from Eq. (2). Then the Lyapunov exponent $\lambda$ is obtained by calculating the limit [13]

$$
\lambda=\lim _{t \rightarrow \infty} \lambda\left(t ; z_{0}, \eta_{0}\right),
$$

where

$$
\lambda\left(t ; z_{0}, \eta_{0}\right)=\frac{1}{t} \ln \left|\eta\left(t ; z_{0}, \eta_{0}\right)\right| .
$$

The finite-time Lyapunov exponent $\lambda\left(t ; z_{0}, \eta_{0}\right)$ depends on the initial conditions $z_{0}$ and $\eta_{0}$. However, assuming ergodicity on the energy-shell, $\lambda$ becomes independent of $z_{0}$, which can then be chosen randomly, e.g., according to the microcanonical distribution. There will also be 
no dependence on initial tangent vectors, because if $\eta_{0}$ is also chosen randomly, it will always have a non-zero component along the most expanding direction. In spite of being redundant, the averaging over $z_{0}$ and $\eta_{0}$ permits to treat equations (2) formally as a system of stochastic differential equations [14]. So, in this "stochastic" approach one attempts the analytical estimation of the average

$$
\lambda=\lim _{t \rightarrow \infty} \frac{1}{t}\left\langle\ln \left|\eta\left(t ; z_{0}, \eta_{0}\right)\right|\right\rangle .
$$

This is a hard task, however. It is much simpler to evaluate the generalized Lyapunov exponent [15, 16]

$$
\lambda_{2}=\lim _{t \rightarrow \infty} \frac{1}{2 t} \ln \left\langle\left|\eta\left(t ; z_{0}, \eta_{0}\right)\right|^{2}\right\rangle,
$$

and assume it approximately coincides with the standard Lyapunov exponent, which is justified in the absence of intermittency [16].

Moreover, if the Hamiltonian of the system can be decomposed as some "free" part plus weak interactions, then perturbative techniques, like the cumulant expansion [14, 17, 18], can be invoked. This is essentially the approach followed by Barnett et al. [19-21], Pettini et al. 22 24], and the present authors 25 27]. Though there are some differences among the formulations of the three groups above, it may be said that the main theoretical conclusion extracted from that body of work is the following. As far as $\lambda_{2}$ is concerned, if one combines the cumulant expansion with some kind of isotropy approximation (which may be fully justified in some cases), the original problem of $6 \mathrm{~N}$ differential equations can be reduced to a system of only two equations for a "representative" single degree of freedom:

$$
\left(\begin{array}{l}
\dot{\eta}_{1} \\
\dot{\eta}_{2}
\end{array}\right)=\left(\begin{array}{cc}
0 & 1 \\
-\kappa(t) & 0
\end{array}\right)\left(\begin{array}{l}
\eta_{1} \\
\eta_{2}
\end{array}\right)
$$

In this kind of mean-field approximation, the "curvature" $\kappa(t)$ is a scalar stochastic process, whose cumulants can be related to the operator cumulants of the Hessian $\mathbf{V}(t)$ (see, e.g., [25]).

The comparison of theoretical results obtained with the cumulant expansion -truncated at the second orderversus numerical simulations has met mixed success. The agreement is very good for some many-particle systems with bounded weak interactions [26, 27] and for the Fermi-Pasta-Ulam system [24]. On the other side, the results for the 1d-XY model [24] and for a dense onecomponent plasma [19, 28] are not so satisfactory.

Anyway, the mentioned tests, which compare theoretical estimates for $\lambda_{2}$ against numerical calculations for $\lambda$, should be taken with some reservations: (a) Pettini et al. did not check if the approximate equality $\lambda \approx \lambda_{2}$ indeed holds 22 24]. Moreover, their theory includes a fitting parameter [the correlation time of the process $\kappa(t)$ ]. Then, it may happen that the theory really agrees with the simulations, or, alternatively, it may be the case of a disagreement that is compensated by a suitable choice of the correlation time. (b) The authors of Refs. 25 27] derived Eq. (8) from first principles (no fitting parameters) and verified numerically that $\lambda \approx \lambda_{2}$ holds in their tests. However, they used a simple ("brute force" 29]) Montecarlo sampling for doing the average (7). And it is known (e.g., 29]) that simple samplings tend to produce wrong estimates of generalized Lyapunov exponents

$$
\lambda_{Q}=\lim _{t \rightarrow \infty} \frac{1}{Q t} \ln \left\langle\left|\eta\left(t ; z_{0}, \eta_{0}\right)\right|^{Q}\right\rangle .
$$

The larger the value of $Q$, the stronger this spurious effect. (Consistently, there are no difficulties in the numerical calculation of the standard $\lambda$, given that $\lambda_{Q} \rightarrow \lambda$ for $Q \rightarrow 0$.)

In conclusion: if one wants to assess the quality of theoretical predictions unabiguously, then it is necessary to develop trustable Montecarlo algorithms for the calculation of $\lambda_{Q}$. We are not aware of the existence of such methods for Hamiltonian many-particle systems. On the other side, an importance-sampling [30] algorithm was recently proposed by Vanneste for calculating $\lambda_{Q}$ in stochastic dynamical systems. The algorithm was shown to perform efficiently for white noise and $Q$ not too large [29].

The present work is part of a larger project that aims at defining the limits of validity of the cumulant approach for the Lyapunov exponent of many-particle Hamiltonian systems. We start our investigations with the simplified mean-field setting (8). This is the simplest possible case having the same formal structure as the many-body problem. By choosing $\kappa(t)$ to be a stochastic process we shall be able to use importance-sampling in the numerical calculations. For several choices of $\kappa(t)$ we shall both analyze the performance of the cumulant expansion and test the numerical algorithms.

It has been argued 24] that, for typical chaotic manybody systems, $\kappa(t)$ should be close to Gaussian white noise; this is the first case we shall consider. For Gaussian white-noise the second-order cumulant expansion for $\lambda_{2}$ is exact, thus this case is ideally suited for analyzing the difficulties that appear in the numerical calculation of $\lambda_{2}$ (Sect. IV).

Next, we keep the Gaussian and Markov properties but allow for finite correlation times, leading to the OrnsteinUhlenbeck process. In this case we calculate the fourth cumulant contribution to $\lambda_{2}$. This test will give us some idea of (i) the convergence rate of the cumulant expansion, and (ii) the performance of the importancesampling method for colored noise (Sect. V).

Last we study the situation of $\kappa(t)$ being Poisson white shot-noise. This appears to be the appropriate choice for modeling the tangent-vector dynamics in dilute gases with short-range interactions. Like in the case of Gaussian white noise, here we have analytical expressions for the generalized exponents $\lambda_{2}, \lambda_{4}, \lambda_{6}$, etc. So, this case will provide an opportunity for further testing of the numerical algorithm. At the same time it will be helpful for characterizing the distribution of finite-time Lyapunov 
exponents, e.g., when is this distribution approximately Gaussian? (Sect. VI).

Section II contains a short review of the cumulant expansion as applied to the determination of some generalized Lyapunov exponents. In Sect. III we describe the three Montecarlo methods considered in this paper: simple, simple-Gaussian, and importance-sampling. Section VII presents a summary of our results and the final remarks.

Before proceeding to the bulk of the paper let us comment that the random oscillator of Eq. (8) is formally equivalent to the Schrödinger equation for a particle in a disordered potential (Anderson localization problem in one dimension). Thus many useful results related to random oscillators can be found in the condensed-matter literature 31 39].

\section{CUMULANT EXPANSION FOR THE KUBO OSCILLATOR}

Equation (8) describes a harmonic oscillator with a random frequency $\omega$ such that $\omega^{2}=\kappa$ (Kubo oscillator). It is worth extending this model a bit to account for the possibility of damping, i.e., we shall consider an oscillator described by the first-order equations

$$
\begin{aligned}
\dot{q} & =p, \\
\dot{p}+\alpha p+\kappa q & =0 .
\end{aligned}
$$

Let us make the identifications $q=\eta_{1}, p=\eta_{2}$ [59]. Then, putting $\alpha=0$ we recover (8).

Some analytical results for the Lyapunov exponent of the Kubo oscillator (10) can be found in the literature (see, e.g., [40-42]). Here we shall concentrate on the generalized exponent $\lambda_{2}$. For this purpose we must consider the dynamics of second order products:

$$
\frac{d}{d t}\left(\begin{array}{c}
q^{2} \\
p^{2} \\
q p
\end{array}\right)=\left(\begin{array}{ccc}
0 & 0 & 2 \\
0 & -2 \alpha & -2 \kappa \\
-\kappa & 1 & -\alpha
\end{array}\right)\left(\begin{array}{c}
q^{2} \\
p^{2} \\
q p
\end{array}\right) \equiv \mathbf{B}(t)\left(\begin{array}{c}
q^{2} \\
p^{2} \\
q p
\end{array}\right)
$$

Let us assume that both parameters $\alpha$ and $\kappa$ are stationary stochastic processes. If fluctuations are small enough (in a sense that will be discussed later), one can obtain dynamical equations for the second-order averages using the cumulant expansion [14]. Splitting the stochastic matrix as an average plus fluctuations:

$$
\mathbf{B}(t)=\mathbf{B}_{\mathbf{0}}+\mathbf{B}_{\mathbf{1}}(t),
$$

it can be shown that for long times one has [14]:

$$
\frac{d}{d t}\left\langle\left(\begin{array}{c}
q^{2} \\
p^{2} \\
q p
\end{array}\right)\right\rangle=\mathbf{K}\left\langle\left(\begin{array}{c}
q^{2} \\
p^{2} \\
q p
\end{array}\right)\right\rangle
$$

where $\mathbf{K}$ is the $3 \times 3$ matrix given by the operator cumulant expansion [14]

$$
\mathbf{K}=\mathbf{B}_{0}+\int_{0}^{\infty}\left\langle\mathbf{B}_{1}(\tau) e^{\mathbf{B}_{0} \tau} \mathbf{B}_{1}(0)\right\rangle e^{-\mathbf{B}_{0} \tau} d \tau+\ldots
$$

Ellipsis stand for third and higher cumulants (some explicit expressions can be found in [18]). The exponent $\lambda_{2}$ is related to the eigenvalue of $\mathbf{K}$ that has the largest real part:

$$
\lambda_{2}=\frac{1}{2} \max \Re\left\{k_{1}, k_{2}, k_{3}\right\},
$$

with $k_{i}$ the eigenvalues of $\mathbf{K}$.

Starting from the evolution equations for higher order products [analogous to (11)] and repeating the same steps above, one can derive the corresponding expressions for $\lambda_{4}, \lambda_{6}$, etc. Of course, the algebraic difficulties increase with the order of the exponent.

\section{NUMERICAL METHODS}

The numerical evolution of Eqs. (10) was performed by means of the Euler algorithm with time step $d t=10^{-3}$ (some higher-order algorithms [43] were tested, but did not lead to substantial improvements). A set of trajectories is generated by randomly choosing $\left(q_{0}, p_{0}\right), \alpha(t)$ and $\kappa(t)$. For each trajectory we computed the norm $|\eta(t)|=\sqrt{q^{2}+p^{2}}$ as a function of time. The Lyapunov exponent is then approximated by the average of finitetime exponents:

$$
\lambda \approx\left\langle\lambda\left(t ; \zeta_{0}\right)\right\rangle=\left\langle\frac{1}{t} \ln \left|\eta\left(t ; \zeta_{0}\right)\right|\right\rangle
$$

The operation $\langle\cdots\rangle$ means averaging over a certain number of realizations of the pseudorandom variables (compactly denoted by $\zeta_{0}$ ) that determine the trajectories. Time $t$ must be large enough to assure the convergence of the average to the desired precision.

In principle we could use the same scheme as before for estimating generalized exponents, i.e.,

$$
\lambda_{Q} \approx \frac{1}{Q t} \ln \left\langle\left|\eta\left(t ; \zeta_{0}\right)\right|^{Q}\right\rangle=\frac{1}{Q t} \ln \left\langle e^{Q t \lambda\left(t ; \zeta_{0}\right)}\right\rangle,
$$

the last equality following from (16). However such a simple averaging tends to undestimate rare events. Hence, spurious results are expected whenever the distribution $P\left(\lambda_{t}\right)$ does not decay fast enough [29, 39, 44]. A somewhat better alternative is, instead of straightforward averaging, to estimate the generalized exponent from the first terms of the series

$$
\lambda_{Q} \approx \sum_{n \geq 1} \frac{(Q t)^{n-1}}{n !} \kappa_{n}(t)
$$

where $\kappa_{n}$ are the $n$ th-order cumulants of $P\left(\lambda_{t}\right)$ [45]. In principle, these cumulants could be estimated numerically. However, for the samples we considered, third and higher cumulants are typically rather unstable [44]. So, it is practically impossible to assess the convergence of the expansion (18). For this reason, cumulants $\kappa_{n}$, with 
$n \geq 3$, will not be included in our calculations. Thus we arrive at

$$
\lambda_{Q} \approx \lambda+\frac{1}{2} Q t \kappa_{2}(t)
$$

[If $P\left(\lambda_{t}\right)$ is Gaussian, this expression is exact.] We call the procedure leading to Eq. (19) simple Gaussian averaging. From Eq. (19) one can derive approximate expressions for the standard Lyapunov exponent, the simplest one being

$$
\lambda \approx 2 \lambda_{2}-\lambda_{4}
$$

Conversely, when $\lambda, \lambda_{2}, \lambda_{4}$ are known, the deviation from equality in the formula above provides a measure of the non-Gaussianity of $P\left(\lambda_{t}\right)$.

When the tail of $P\left(\lambda_{t}\right)$ is essential for the determination of $\lambda_{Q}$ and it is not Gaussian, the approximations (17) and (19) are bound to fail. In this case one must resort to numerical methods capable of sampling the relevant part of the distribution $P\left(\lambda_{t}\right)$. The importance-sampling Monte Carlo algorithm recently proposed by Vanneste is especially suited for our needs. The algorithm, both efficient and easy to implement, uses a simple random resampling step: those trajectories which contribute the most (least) to the average are cloned (pruned) with a large probability [29].

Having presented the theory and the numerical methods, we are ready to proceed with the comparisons.

\section{GAUSSIAN WHITE NOISE}

Only when the matrix stochastic process $\mathbf{B}_{\mathbf{1}}$ is Gaussian and delta-correlated the cumulant expansion does stop at the second order, i.e., Eq. (14) without the ellipsis becomes exact [18]. This is the case we consider now.

(Stochastic differential equations with multiplicative white noise will always be taken in the Stratonovich sense.)

\section{A. Random frequency}

Let us first study the situation where the damping $\alpha$ is a constant and

$$
\kappa(t)=\kappa_{0}+\xi(t)
$$

where $\xi(t)$ is a zero-mean Gaussian white noise with correlation function

$$
\left\langle\xi(t) \xi\left(t^{\prime}\right)\right\rangle=\Delta \delta\left(t-t^{\prime}\right) .
$$

With these definitions one has

$$
\mathbf{B}=\left(\begin{array}{ccc}
0 & 0 & 2 \\
0 & -2 \alpha & -2 \kappa_{0} \\
-\kappa_{0} & 1 & -\alpha
\end{array}\right)+\xi(t)\left(\begin{array}{lll}
0 & 0 & 0 \\
0 & 0 & 2 \\
1 & 0 & 0
\end{array}\right)
$$

After substitution into Eq. (14) we readily obtain

$$
\mathbf{K}=\left(\begin{array}{ccc}
0 & 0 & 2 \\
\Delta & -2 \alpha & -2 \kappa_{0} \\
-\kappa_{0} & 1 & -\alpha
\end{array}\right)
$$

The generalized exponent $\lambda_{2}$ can now be calculated from Eq. (15). A closed expression for the standard Lyapunov exponent was derived by Mallick and Peyneau [40]. As an example, we display in Fig. 1 analytical and numerical results for both exponents. Given that the theoretical

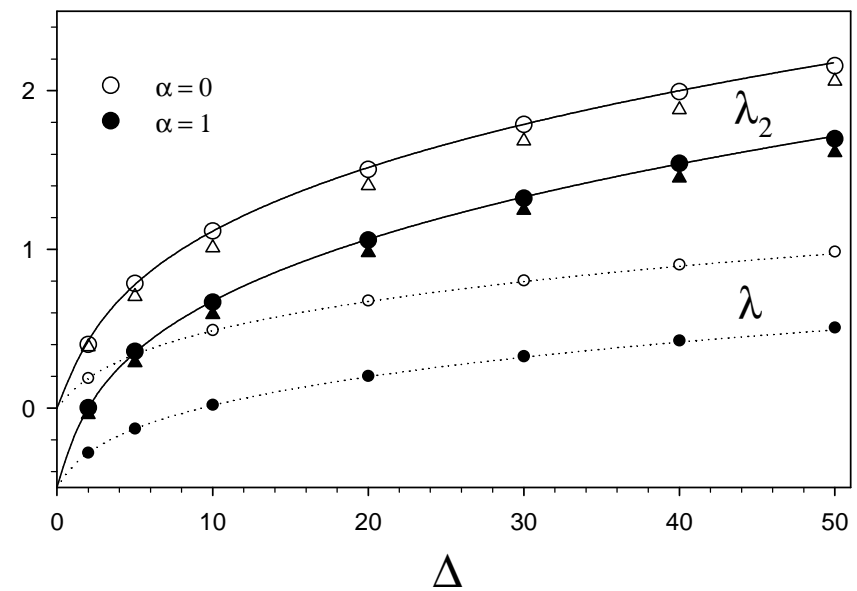

FIG. 1: Lyapunov exponents versus noise strength for the harmonic oscillator with random frequency. Symbols correspond to numerical results for $\lambda$ (small circles) and $\lambda_{2}$ (large circles: importance-sampling; triangles: simple Gaussian sampling). Two values of the damping constant were used: $\alpha=0$ (hollow symbols) and $\alpha=1$ (filled symbols). In both cases $\kappa_{0}=1$. In all cases we averaged over $10^{3}$ trajectories. Resampling time was set to $t_{\text {res }}=1.0$. Lines correspond to exact theoretical expressions.

expressions are exact, this comparison constitutes a rigorous test for the numerical methods. We see that, even for relatively small samples, the importance-sampling calculation agrees perfectly with the theory. The Gaussian sampling, though not perfect, provides a reasonably good approximation.

Clearly both exponents, $\lambda_{2}$ and $\lambda$, do not coincide. This is to be expected whenever fluctuations in the frequency/damping are large as compared to their average values [35, 45].

The higher order exponents $\lambda_{2 J}$, with $J=2,3, \ldots$ are obtained by diagonalizing matrices of size $2 J-1$. Such matrices describe the evolution of the moments $\left\langle q^{n} p^{m}\right\rangle$, with $m+n=2 J$, and have simple analytical expressions [35, 45]. An example involving a higher order exponent will be shown in Sec. VI 


\section{B. Random damping}

Now we consider a harmonic oscillator with constant frequency but in an environment with fluctuating damping coefficient

$$
\alpha(t)=\alpha_{0}+\xi(t)
$$

where $\xi(t)$ is again zero-mean Gaussian white noise, with correlation given by Eq. (22).

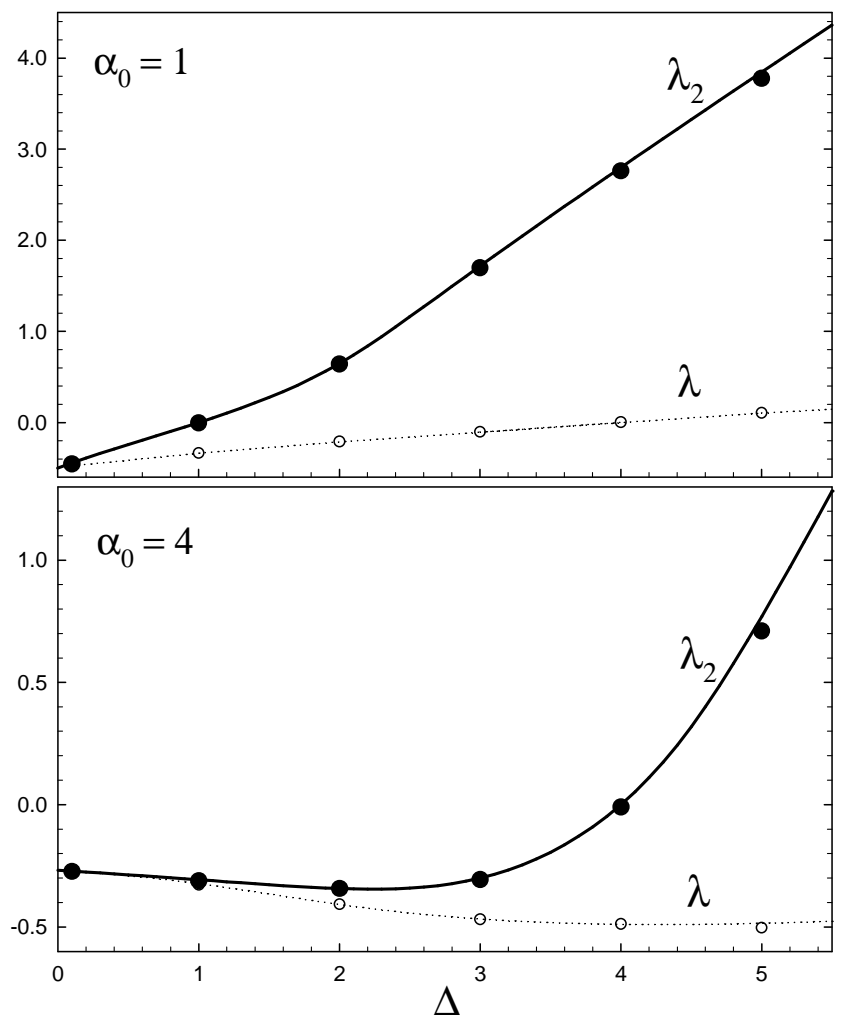

FIG. 2: Lyapunov exponents vs. noise strength for the harmonic oscillator with random damping. Lines represent exact theoretical results. Symbols correspond to numerical calculations for $\lambda_{2}$ (large circles) and $\lambda$ (small circles). Importancesampling Monte Carlo was used in the case of $\lambda_{2}$. We chose two values for the average damping coefficient: $\alpha_{0}=1$ (top panel), $\alpha_{0}=4$ (bottom panel). In both cases $\kappa=1$. In all cases we averaged over $10^{3}$ trajectories. Resampling time was set to $t_{\text {res }}=0.2$.

Now the matrix B is decomposed as

$$
\mathbf{B}=\left(\begin{array}{ccc}
0 & 0 & 2 \\
0 & -2 \alpha_{0} & -2 \kappa \\
-\kappa & 1 & -\alpha_{0}
\end{array}\right)+\xi(t)\left(\begin{array}{lll}
0 & 0 & 0 \\
0 & 2 & 0 \\
0 & 0 & 1
\end{array}\right)
$$

Hence, substitution into (14) yields

$$
\mathbf{K}=\left(\begin{array}{ccc}
0 & 0 & 2 \\
\Delta & -2 \alpha_{0}+2 \Delta & -2 \kappa \\
-\kappa & 1 & -\alpha_{0}+\Delta / 2
\end{array}\right)
$$

Upon diagonalizing $\mathbf{K}$ we obtain $\lambda_{2}$. Figure 2 presents the comparison of numerical and analytical results for $\lambda$ and $\lambda_{2}$ as the noise intensity is varied (exact theoretical results for $\lambda$ were extracted from Ref. [41]).

Concerning the numerical calculation of $\lambda_{2}$, besides noting the excellent agreement with the theory, it must be said that the importance-sampling method behaved in a very robust way both for random frequency and random damping. Changing sample size, simulation time, and resampling time $t_{\text {res }}$ [29] within reasonable limits did not appreciably affect the result for $\lambda_{2}$. However, if one increases $t_{\text {res }}$ beyond certain bounds, then the method becomes inefficient, as very large samples are necessary to guarantee convergence to the correct results.

\section{CORRELATED NOISE}

For white noise fluctuations, either in the frequency or in the damping, we have verified in the previous section that the theory for $\lambda_{2}$ is in agreement with numerical results, provided the latter are obtained using importance sampling.

Now we shall analyze the effect of introducing noise correlations. We consider the case of a random frequency, as in Eq. (21), but now the noise is a zero-mean OrnsteinUlhenbeck process, i.e., with correlation function

$$
\left\langle\xi(t) \xi\left(t^{\prime}\right)\right\rangle=\frac{\Delta}{2 \tau} \exp \left(-\left|t-t^{\prime}\right| / \tau\right) \equiv \sigma^{2} \exp \left(-\left|t-t^{\prime}\right| / \tau\right) .
$$

For simplicity we set $\alpha=0$ and $\kappa_{0}=0$. By inserting (23) into (14), the second-cumulant matrix becomes

$$
\mathbf{K}^{(2)}=\left(\begin{array}{ccc}
0 & 0 & 2 \\
\Delta & -2 \Delta \tau^{2} & 0 \\
\Delta \tau & 1 & -2 \Delta \tau^{2}
\end{array}\right)
$$

Notice that in the limit $\tau \rightarrow 0$ the white-noise case is recovered.

In the presence of correlations the second-order truncation of the cumulant expansion (14) is not exact. In order to improve the theory one must calculate higher cumulants. For the present case the third cumulant is null. Explicit expressions for the fourth cumulant were given by Fox [18], Breuer et al. [46], and Tessieri [34]. A somewhat lengthy calculation (sketched in Appendix A leads to the following result for the fourth order approximation to $\mathbf{K}$ :

$$
\mathbf{K}^{(4)}=\mathbf{K}^{(2)}+\frac{1}{2} \Delta^{2} \tau^{3}\left(\begin{array}{ccc}
0 & 0 & 0 \\
13 & 74 \tau^{2} & -57 \tau \\
17 \tau & 173 \tau^{3} & -99 \tau^{2}
\end{array}\right)
$$

The comparison between numerical and theoretical results for $\lambda_{2}$ is presented in Fig. 3. For completeness we also show numerical calculations of the standard Lyapunov exponent, together with an approximate theoretical expression obtained along the lines of Ref. [40] (see Appendix B]). 


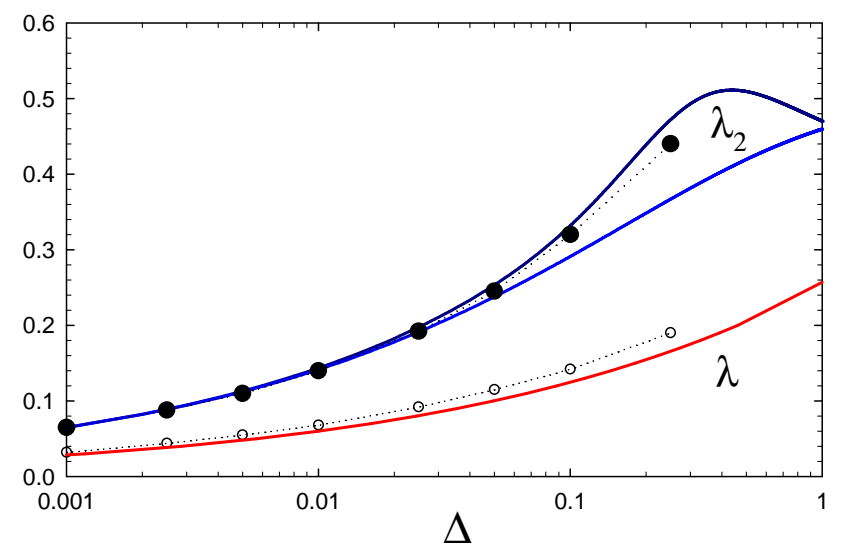

FIG. 3: (Color online.) Harmonic oscillator with correlated random frequency. Symbols indicate numerical results for $\lambda_{2}$ (full circles) and $\lambda$ (hollow circles) as a function of the noise amplitude $\Delta$ (averages over $10^{4}$ trajectories). Parameters are $\alpha=0, \kappa_{0}=0$ and $\tau=1$. Resampling time was set to $t_{\text {res }}=$ 20. Solid lines correspond to theoretical results. For $\lambda_{2}$ we used the cumulant expansion (blue: truncation at the second cumulant; dark blue: including the fourth cumulant). An approximate analytical expression for $\lambda$ (red) is also shown.

We see in Fig. 3 that the inclusion of the fourth cumulant contribution noticeably extends the domain of validity of the theory into the region of larger noise amplitudes (with respect to the second-order approximation). Higher cumulants can also be calculated, but the required effort quickly becomes unbearable. For instance, the sixth cumulant demands the calculation of more than 100 terms (see Appendix A). Anyway, the theory being perturbative, by increasing the amplitude of the noise and/or the correlation time, one eventually arrives at a point were the cumulant expansion completely breaks down.

The perturbation parameter controlling the convergence of the cumulant expansion is the so-called Kubo number $\varepsilon$. General considerations led van Kampen [14] to conclude that the Kubo number is the product of the amplitude of the fluctuations and the correlation time, that is $\sigma \tau$. However, in the present case it is clear that such a combination is not adimensional. The correct Kubo number is instead

$$
\varepsilon=\sigma \tau^{2}=\sqrt{\frac{\Delta \tau^{3}}{2}}
$$

This can be checked explicitly from the second and fourth cumulants above. Consider, for instance, the element $\mathbf{K}_{21}$, which dominates the Lyapunov exponent for small correlation times:

$$
\mathbf{K}_{21}=\Delta+\frac{13}{2} \Delta^{2} \tau^{3}+\cdots=\Delta\left(1+\frac{13}{2} \Delta \tau^{3}+\ldots\right) .
$$

In the white-noise limit, i.e., $\tau \rightarrow 0$ with $\Delta$ fixed, the Kubo number tends to zero -as it should be.
On the numerical side, we comment that for large noise amplitudes the convergence to the limiting values is much slower than in the white-noise cases. The points in Fig. 3 were obtained by a double limiting procedure. For a fixed resampling time $t_{\text {res }}$, we increased the number of samples until convergence was reached. Then we iterated the scheme for increasing values of $t_{\text {res }}$ until a stable value for $\lambda_{2}$ was obtained. The larger the resampling time, the larger the number of samples to keep the error within the chosen bounds.

\section{POISSON SHOT NOISE}

In a dilute gas with short-range interactions, phasespace coordinates evolve trivially in-between collisions. During collisions, positions remain essentially unchanged while momenta experience sudden jumps. The same description applies to tangent-space coordinates. Thus, in a mean-field setting, the tangent dynamics of a representative (effective) particle is described by Eq. (8), the stochastic frequency corresponding to Poisson shot noise: [47 54]

$$
\kappa(t)=\sum_{i} A_{i} \delta\left(t-t_{i}\right)
$$

Neglecting correlations among collisions the amplitudes $A_{i}$ will be modeled by independent stochastic variables (identically distributed). Accordingly, the succession of collision times $\left\{t_{i}\right\}$ constitutes a Poisson process.

The random oscillator (8) with Poisson frequency (33) was solved by van Kampen [52] (including damping and additive noise). He derived an exact integro-differential equation for the probability distribution $P(q, p, t)$ from where the evolution of the moments $\left\langle q^{n} p^{m}\right\rangle$ can be sistematically obtained [52]. For the second moments one gets

$$
\frac{d}{d t}\left(\begin{array}{c}
\left\langle q^{2}\right\rangle \\
\left\langle p^{2}\right\rangle \\
\langle q p\rangle
\end{array}\right)=\left(\begin{array}{ccc}
0 & 0 & 2 \\
\rho\left\langle A^{2}\right\rangle & 0 & -2 \rho\langle A\rangle \\
-\rho\langle A\rangle & 1 & 0
\end{array}\right)\left(\begin{array}{c}
\left\langle q^{2}\right\rangle \\
\left\langle p^{2}\right\rangle \\
\langle q p\rangle
\end{array}\right)
$$

where $\rho$ is the collision frequency.

Remarkably the expression above can be shown to coincide with the result of the second-order cumulant approach (13/14). However, the higher-order cumulants of $\kappa(t)$ are not null, rather, they are delta-correlated $50-$ 52]. Just they do not affect the asymptotic growth of the second moments.

The equations for the fourth moments $\left\langle q^{4}\right\rangle,\left\langle p^{4}\right\rangle$, $\left\langle q^{2} p^{2}\right\rangle,\left\langle q^{3} p\right\rangle,\left\langle q p^{3}\right\rangle$ can also be calculated without much effort. The corresponding matrix reads

$$
\left(\begin{array}{ccccc}
0 & 0 & 0 & 4 & 0 \\
\rho\left\langle A^{4}\right\rangle & 0 & 6 \rho\left\langle A^{2}\right\rangle & -4 \rho\left\langle A^{3}\right\rangle & -4 \rho\langle A\rangle \\
\rho\left\langle A^{2}\right\rangle & 0 & 0 & -2 \rho\langle A\rangle & 2 \\
-\rho\langle A\rangle & 0 & 3 & 0 & 0 \\
-\rho\left\langle A^{3}\right\rangle & 1 & -3 \rho\langle A\rangle & 3 \rho\left\langle A^{2}\right\rangle & 0
\end{array}\right)
$$


from where one extracts the fourth-order generalized exponent $\lambda_{4}$.

Figure 4 shows that, when importance-sampling is used, the agreement between theory and numerics is excellent. On the other side, simple sampling (plus a Gaussian approximation) leads to deviations from the theory, which become stronger as collision frequency ("density") is lowered. Of course this disagreement is a consequence of the nonGaussianity of the distribution of finite-time Lyapunov exponents, and can also be observed when comparing $\lambda$ vs $2 \lambda_{2}-\lambda_{4}$ (this can be thought of as a failure of the replica trick [16, 55] in its crudest version).

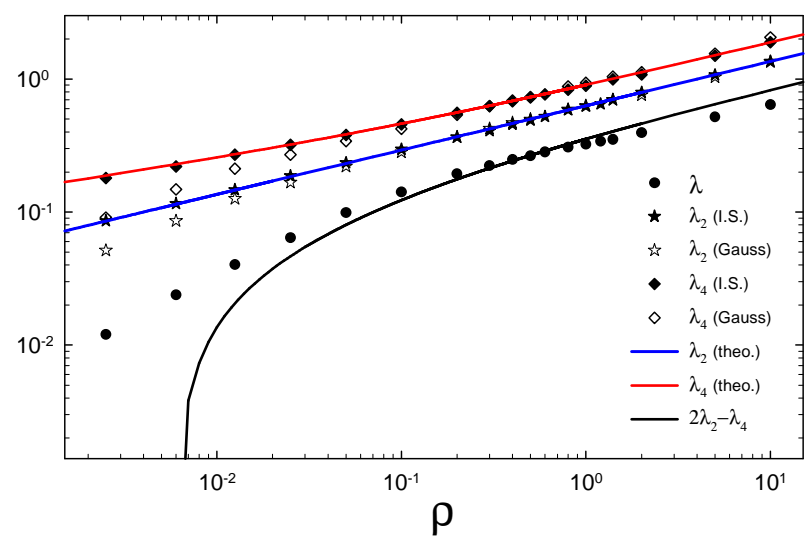

FIG. 4: (Color online.) Harmonic oscillator with Poissonshot-noise frequency. We show the Lyapunov exponents $\lambda$, $\lambda_{2}$ and $\lambda_{4}$ as a function of collision frequency $\rho$. Red/blue lines indicate theoretical estimates for $\lambda_{4} / \lambda_{2}$, and the corresponding symbols stand for numerical calculations using either simple-Gaussian sampling (open symbols) or importancesampling (full symbols). Shown is also the theoretical result for $2 \lambda_{2}-\lambda_{4}$ (black line), which is an estimate of the standard Lyapunov exponent $\lambda$ (circles, numerical).

The numerical method worked satisfactorily, the relation between parameter values and efficiency being similar to the white-noise cases analyzed in Sect. IV.

\section{FINAL REMARKS}

We analysed the random harmonic oscillator as a simplified model of the tangent dynamics of many-particle systems. In spite of its relative simplicity, this model already exhibits some of the essential features and characteristics of high-dimensional systems.

Specifically, we were able to assess the performance of the importance-sampling approach for the numerical calculation of generalized Lyapunov exponents. In all the considered cases -some of which unaccessible by standard sampling methods- we confirmed that the method works satisfactorily, and developed some intuition about the appropriate values of the parameters (i.e., resampling time and number of samples) that result in a faster convergence.

On the theoretical side, we carried out several tests of the cumulant approach in nontrivial cases, i.e., for frequencies corresponding to Ornstein-Uhlenbeck and Poisson processes. In particular, we identifyed the correct perturbative parameter (Kubo number) and -not unsurprisingly - verified that the second-order truncation of the cumulant series gives the exact second-order generalized exponent $\lambda_{2}$ for the case of Poisson shot noise.

Concerning the application of the cumulant approach to dilute gases, we note that in this case the tangent dynamics can be thought to be driven by multivariate Poisson noise. Accordingly the second-order truncation could indeed produce the exact $\lambda_{2}$-like in the one-dimensional problem. However, the verification of this expectation would require the numerical calculation of $\lambda_{2}$ for a Hamiltonian, i.e., nonstochastic, system. In order to implement an importance-sampling algorithm for this case one should somehow introduce noise in the dynamics, then calculate $\lambda_{2}$ as a function of the noise intensity, and extrapolate the results to zero noise 58]. Several ideas for constructing such an algorithm are currently under investigation.

\section{Acknowledgements:}

We acknowledge Brazilian agencies Faperj and $\mathrm{CNPq}$ for partial financial support.

\section{Appendix A: Fourth cumulant}

Here we briefly describe the calculation of the fourthcumulant contribution to the generalized Lyapunov exponent of the Ornstein-Uhlenbeck oscillator, i.e., the rightmost term in Eq. (30). In general, this contribution reads: [18, 34, 46]

$$
K_{4}(t) \equiv \mathbf{K}^{(4)}-\mathbf{K}^{(2)}=e^{B_{0} t} Q_{4}(t) e^{-B_{0} t},
$$

where

$$
\begin{aligned}
Q_{4}(t)= & \int_{0}^{t} d t_{1} \int_{0}^{t_{1}} d t_{2} \int_{0}^{t_{2}} d t_{3} \\
& \left(\left\langle\tilde{B}_{1}(t) \tilde{B}_{1}\left(t_{1}\right) \tilde{B}_{1}\left(t_{2}\right) \tilde{B}_{1}\left(t_{3}\right)\right\rangle\right. \\
& -\left\langle\tilde{B}_{1}(t) \tilde{B}_{1}\left(t_{1}\right)\right\rangle\left\langle\tilde{B}_{1}\left(t_{2}\right) \tilde{B}_{1}\left(t_{3}\right)\right\rangle \\
& -\left\langle\tilde{B}_{1}(t) \tilde{B}_{1}\left(t_{2}\right)\right\rangle\left\langle\tilde{B}_{1}\left(t_{1}\right) \tilde{B}_{1}\left(t_{3}\right)\right\rangle \\
& \left.-\left\langle\tilde{B}_{1}(t) \tilde{B}_{1}\left(t_{3}\right)\right\rangle\left\langle\tilde{B}_{1}\left(t_{1}\right) \tilde{B}_{1}\left(t_{2}\right)\right\rangle\right)
\end{aligned}
$$

with

$$
\tilde{B}_{1}(t)=e^{-B_{0} t} B_{1}(t) e^{B_{0} t}
$$


For the large times we are interested in, i.e., $t \gg \tau, K_{4}(t)$ becomes time independent. Next we note that matrices $\tilde{B}_{1}$ are proportional to the scalar Ornstein-Ulhenbeck process $\xi(t)$. So, one must only calculate two- and fourtime correlators of $\xi(t)$. By virtue of the Gaussian property [56], the four-time correlator is expressible as a sum of products of two-time functions (28). Finally one calculates the triple integrals and takes the limit $t \rightarrow \infty$ (with the help of an appropriate software, e.g., Mathematica [57]), arriving thus at the desired result (30).

\section{Appendix B: Lyapunov exponent}

Here we sketch the steps leading to the approximate expression for the Lyapunov exponent (of the randomfrequency Ornstein-Ulhenbeck oscillator) that is plotted in Fig. 3. We have simply adapted the calculations of Mallick and Peyneau [40] to the case $\kappa_{0}=0$.

In the absence of damping, the Lyapunov exponent can be obtained as [40]

$$
\begin{aligned}
\lambda & =\lim _{t \rightarrow \infty} \frac{1}{2 t}\left\langle\ln \left(q^{2}+\dot{q}^{2}\right)\right\rangle \\
& =\lim _{t \rightarrow \infty} \frac{1}{2} \frac{d}{d t}\left\langle\ln \left(q^{2}+\dot{q}^{2}\right)\right\rangle \\
& =\lim _{t \rightarrow \infty}\left(\langle y\rangle+\frac{1}{2} \frac{d}{d t}\left\langle\ln \left(y^{2}+1\right)\right\rangle\right) \\
& =\lim _{t \rightarrow \infty}\langle y\rangle,
\end{aligned}
$$

where $y=\dot{q} / q$. From Eq. (8) one sees that $y$ obeys the following nonlinear equation

$$
\dot{y}=-y^{2}+\eta(t) \text {. }
$$

We will first find the exact expression for $\lambda$ when the noise is white (intensity $\Delta$ ). In this case, the associated Fokker-Planck equation for $P(y, t)$, i.e.,

$$
\partial_{t} P=\partial_{y}\left(y^{2} P\right)+\frac{\Delta}{2} \partial_{y y} P
$$

has the following steady state solution:

$$
P_{\mathrm{ss}}(y)=N e^{-2 y^{3} /(3 \Delta)} \int_{-\infty}^{y} e^{2 x^{3} /(3 \Delta)} d x
$$

where $N$ is a normalization constant. By averaging over the steady state we obtain

$$
\lambda^{\mathrm{w}}(\Delta)=\int_{-\infty}^{\infty} d y \text { y } P_{\mathrm{ss}}(y)=\frac{\sqrt{\pi}}{\Gamma\left(\frac{1}{6}\right)}\left(\frac{3 \Delta}{4}\right)^{\frac{1}{3}} \simeq 0.2893 \Delta^{1 / 3} .
$$

In the case of an arbitrary correlation time $\tau$, by using a mean-field approximation ("decoupling ansatz" [40]), one can derive the following equation for $\lambda$ :

$$
\lambda(\Delta, \tau) \simeq \lambda^{\mathrm{w}}\left(\frac{\Delta}{1+2 \tau \lambda(\Delta, \tau)}\right) .
$$

So, the final result comes in the form of an implicit equation:

$$
\lambda(\Delta, \tau) \simeq 0.289\left(\frac{\Delta}{1+2 \tau \lambda(\Delta, \tau)}\right)^{\frac{1}{3}}
$$

This approximate relation slightly underestimates the numerical results of Fig. 3 .
[1] N. S. Krylov, Works on the Foundations of Statistical Physics (Princeton University Press, Princeton, 1979).

[2] S.-K. Ma, Statistical Mechanics (World Scientific, Singapore, 1985).

[3] Y. G. Sinai, Russ. Math. Surv. 25, 137 (1970).

[4] H. van Beijeren and J. R. Dorfman, Phys. Rev. Lett. 74, 4412 (1995).

[5] H. van Beijeren, A. Latz, and J. R. Dorfman, Phys. Rev. E 57, 4077 (1998).

[6] R. van Zon, H. van Beijeren, and Ch. Dellago, Phys. Rev. Lett. 80, 2035 (1998).

[7] H. V. Kruis, D. Panja, and H. van Beijeren, J. Stat. Phys. 124, 823 (2006).

[8] J. R. Dorfman, An Introduction to Chaos in Nonequilibrium Statistical Mechanics (Cambridge University Press, Cambridge, UK, 1999).

[9] Ch. Dellago, H. A. Posch, and W. G. Hoover, Phys. Rev. E 53, 1485 (1996).

[10] Ch. Dellago, H. A. Posch, Physica A 240, 68 (1997).

[11] J. C. Kimball, Phys. Rev. E 63, 066216 (2001).

[12] P. V. Elyutin, Phys. Lett. A 331, 153 (2004).
[13] G. Benettin, L. Galgani, and J.-M. Strelcyn, Phys. Rev. A 14, 2338 (1976).

[14] N. G. van Kampen, Stochastic Processes in Physics and Chemistry (North-Holland, Amsterdam, 1981).

[15] R. Benzi, G. Paladin, G. Parisi, and A. Vulpiani, J. Phys. A 18, 2157 (1985).

[16] P. Castiglione, M. Falcioni, A. Lesne, and A. Vulpiani, Chaos and Coarse Graining in Statistical Mechanics (Cambridge University Press, New York, 2008).

[17] R. Kubo, J. Phys. Soc. Japan 17, 1100 (1962).

[18] R. F. Fox, J. Math. Phys 15, 1479 (1974).

[19] D. M. Barnett, T. Tajima, K. Nishihara, Y. Ueshima, and H. Furukawa, Phys. Rev. Lett. 76, 1812 (1996).

[20] Y. Ueshima, K. Nishihara, D. M. Barnett, T. Tajima, and H. Furukawa, Phys. Rev. E 55, 3439 (1997).

[21] D. M. Barnett and T. Tajima, Phys. Rev. E 54, 6084 (1996).

[22] L. Casetti, R. Livi, and M. Pettini, Phys. Rev. Lett. 74, 375 (1995).

[23] L. Casetti, C. Clementi, and M. Pettini, Phys. Rev. E 54, 5969 (1996). 
[24] L. Casetti, M. Pettini, and E. G. D. Cohen, Phys. Rep. 337, 238 (2000).

[25] R. O. Vallejos and C. Anteneodo, Phys. Rev. E 66, 021110 (2002).

[26] C. Anteneodo, R. N. P. Maia, and R. O. Vallejos, Phys. Rev. E 68, 036120 (2003).

[27] R.O. Vallejos and C. Anteneodo, Physica A 340, 178 (2004).

[28] A. Torcini, Ch. Dellago, and H. A. Posch, Phys. Rev. Lett. 83, 2676 (1999); D. M. Barnett, T. Tajima, and Y. Ueshima, ibid. 83, 2677 (1999).

[29] J. Vanneste, Phys. Rev. E 81036701 (2010).

[30] K. Binder and D. W. Heermann, Monte Carlo Simulation in Statistical Physics: An Introduction (Springer-Verlag, Berlin, 2010).

[31] G. Paladin and A. Vulpiani, Phys. Rep. 56, 147 (1987).

[32] L. Tessieri and F. M. Izrailev, Phys. Rev. E 62, 3090 (2000).

[33] L. Tessieri and F. M. Izrailev, Phys. Rev. E 64, 066120 (2001).

[34] L. Tessieri, J. Phys. A: Math. Gen. 35, 9585 (2002).

[35] H. Schomerus and M. Titov, Phys. Rev. E 66, 066207 (2002).

[36] E. Gurevich and O. Kenneth, Phys. Rev. A 79, 063617 (2009).

[37] P. Lugan, A. Aspect, L. Sanchez-Palencia, D. Delande, B. Grémaud, C. A. Müller, and C. Miniatura, Phys. Rev. A 80, 023605 (2009).

[38] A. Iomin, Phys. Rev. E 79, 062102 (2009).

[39] E. Gurevich and A. Iomin, Phys. Rev. E 83, 011128 (2011).

[40] K. Mallick and P. E. Peyneau, Physica D 221, 72 (2006).

[41] N. Leprovost, S. Aumaître, K. Mallick, Eur. Phys. J. B 49, 453 (2006).

[42] Y. Peleg and E. Barkai, Phys. Rev. E 80, 030104R (2009).
[43] P. E. Kloeden and E. Platen, Numerical Solution of Stochastic Differential Equations (Springer-Verlag, Berlin, 1992).

[44] C. Anteneodo and R. O. Vallejos, J. Phys. Conf. Ser. 246, 012002 (2010).

[45] R. Zillmer and A. Pikovsky, Phys. Rev. E 67, 061117 (2003).

[46] H.-P. Breuer, A. Ma, and F. Petruccione, preprint arXiv:quant-ph/0209153v1 (2002).

[47] P. Hänggi, Phys. Lett. 78A, 304 (1980).

[48] P. Hänggi, Z. Phys. B 36, 271 (1980).

[49] P. Hänggi, Z. Phys. B 43, 269 (1981).

[50] K. Lindenberg, V. Seshadri, and B. J. West, Phys. Rev. A 22, 2171 (1980).

[51] B. J. West, K. Lindenberg, and V. Seshadri, Physica 102A, 470 (1980).

[52] N. G. van Kampen, Physica 102A, 489 (1980).

[53] C. van den Broeck, J. Stat. Phys. 31, 467 (1983).

[54] K. Lindenberg and B. J. West, The Nonequilibrium Statistical Mechanics of Open and Closed Systems (WileyVCH, New York, 1990).

[55] M. J. de Oliveira and A. Petri, Phys. Rev. 53, 2960 (1996).

[56] R. F. Fox, Phys. Rep. 48, 179 (1978).

[57] S. Wolfram, Mathematica: A System for Doing Mathematics by Computer, 2nd ed. (Addison-Wesley, Reading, MA, 1991).

[58] C. Giardinà, J. Kurchan, and L. Peliti, Phys. Rev. Lett. 96, 120603 (2006).

[59] The equations of motion of the random harmonic oscillator being linear, phase space and tangent space can be identified. Accordingly the Lyapunov exponent equals one half the average rate of energy growth. 